\title{
NEW SCHEME FOR EXTRACTING MOLECULAR DYNAMICS FROM SPECTRA: CASE STUDY ON VIBRATIONALLY HIGHLY EXCITED ACETYLENE
}

\author{
KAORU YAMANOUCHI, JUN MIYAWAKI, and SOJI TSUCHIYA \\ Department of Pure and Applied Sciences, College of Arts and Sciences, The \\ University of Tokyo, Komaba, Meguro-ku, Tokyo 153 Japan \\ DAVID M. JONAS \\ Department of Chemistry, The University of Chicago, 5735 S. Ellis Avenue, \\ Chicago, Illinois 60637, U.S.A \\ ROBERT W. FIELD \\ Department of Chemistry, Massachusetts Institute of Technology, Cambridge, \\ Massachusetts 02139, U.S.A.
}

(Received 26 May, 1993)

\begin{abstract}
Three types of analyses: generalized conventional spectroscopic analysis, statistical analysis, and hierarchical analysis, by which we aim to extract dynamical information from the complex spectrum of small polyatomic molecules are reviewed by referring to recent studies on vibrationally highly excited acetylene. A convolution analysis, one type of hierarchical analysis, is performed on the dispersed fluorescence spectrum of acetylene to demonstrate its practical applicability. Based on previous studies, as well as on the convolution analysis, a systematic and efficient procedure to treat such complex spectra is discussed.
\end{abstract}

KEY WORDS: Highly excited vibrational levels, high resolution spectroscopy, molecular dynamics, acetylene, hierarchical analysis, statistical analsysis

\section{INTRODUCTION}

One of the crucial problems for modern molecular spectroscopy is to find an appropriate and efficient procedure to extract dynamical information characteristic of a molecular system in the chemically significant energy region. As demonstrated for $\mathrm{SO}_{2}{ }^{1-3}$ and acetylene, ${ }^{3-14}$ recently developed laser spectroscopic techniques reveal that the level structure of a small polyatomic molecules in a highly excited energy region can be very complex, but it appears possible to deduce information about the dynamical behavior of the system from dense and complex spectra. In this article we briefly 
review three types of analyses by which we aim to extract molecular dynamics from an observed complex spectrum by referring recent studies on vibrationally highly excited acetylene. These three types of analyses are (i) generalized conventional spectroscopic analysis, (ii) statistical analysis, and (iii) hierarchical analysis. In the following sections (Sec. II-IV), we explain how these methods have been applied to vibrationally highly excited acetylene, and in Sec. V, we describe a systematic and efficient procedure for the analysis of the complex observed spectrum by introducing recent studies of the complex laser induced fluorescence (LIF) spectrum of $\mathrm{NO}_{2}$.

\section{GENERALIZED CONVENTIONAL SPECTROSCOPY}

\section{Search for Feature States and Good Quantum Numbers}

If the observed spectrum of a molecular system is a simple one, we can easily assign a set of quantum numbers to each peak in the spectrum based on a Dunham type polynomial formula for the level energies or their numerical values derived from a well-established Hamiltonian. However, as demonstrated for the dispersed fluorescence (DF) spectrum of acetylene, ${ }^{3,4}$ the assignment of vibrational quantum numbers at high vibrational energy becomes far more difficult due to the anharmonic resonances which can be treated easily as a small perturbation at low vibrational energy. Because the off-diagonal elements of an anharmonic coupling, in which the quantum numbers $\mathrm{v}_{\mathrm{j}}$ change by $\mathrm{n}_{\mathrm{j}}$ units, scale roughly as $\Pi\left(\mathrm{v}_{\mathrm{j}}\right)^{(\mathrm{nj} / 2)}$, these anharmonic couplings, which shift the level energy only slightly from their unperturbed position at lower energy, can cause formidably complex level structure and intensity distributions at higher energy.

In the observed DF spectrum of acetylene, the lower energy region (below $14000 \mathrm{~cm}^{-1}$ ) contained six progressions in even quanta of $v_{4}^{\prime \prime}$ (trans-bend) in combination with $v_{2}^{\prime \prime}$ (CC stretch), which were easily identified. However, in the high energy region (above $14000 \mathrm{~cm}^{-1}$ ), the assignments of individual peaks becomes difficult due to the increasing peak-density and spectral complexity. It was expected that the source of the complexity could be clarified by extending secure vibrational assignments from the lower energy region into the highly excited region.

The identification of three additional trans-bend progressions (below $14000 \mathrm{~cm}^{-1}$ ) built on excitations in a third vibrational mode played a key role in untangling the complexity in the spectrum at higher energy. A precise examination of the intensity enhancement observed in these additional progressions and the predictions of the level energy from previously well-determined spectroscopic constants led to the conclusion that a previously undetected $\Delta \mathrm{v}_{4}=-\Delta_{\mathrm{v} 5}= \pm 2$ Darling-Dennison (DD) resonance between the two degenerate bending modes, $v_{4}{ }^{\prime \prime}$ (trans-bend) and $v_{5}{ }^{\prime \prime}$ (cisbend), was the perturbation responsible for inducing a complex spectrum at high energy.

It was argued that, at higher energy, energetically adjacent levels forming a tier of the DD resonances merge with each other and the DD resonance polyad by which 
$\left(\mathrm{v}_{\text {bend }} / 2\right)+1$ levels are connected by $\left(\mathrm{v}_{\text {bend }} / 2\right)$ resonances is produced, where $\mathrm{v}_{\text {bend }}$ represents the total bending quanta, $v_{4}{ }^{\prime \prime}+v_{5}{ }^{\prime \prime}$. Within this tier, both of the quantum numbers $\mathrm{v}_{4}$ " and $\mathrm{v}_{5}{ }^{\prime \prime}$ representing individual degenerate bending modes lose its significance, and only the sum of the two values, $v_{\text {bend }}$, can be used to label the entire $\left(\mathrm{v}_{\text {bend }} / 2\right)+1$ levels in a polyad. In other words, the bright character of the unperturbed state is shared by the levels within the polyad, and we assign a set of vibrational quantum numbers, which represent the zero-order Franck-Condon (FC) bright level to a group of levels. By performing this generalized assignment, the complex level structure above $14000 \mathrm{~cm}^{-1}$ was interpreted reasonably. Thus, even in such a complex spectrum, it was shown that the assignment of a set of vibrational quantum numbers to a group of eigenstate is possible by examining the effect of anharmonic resonances gradually ascending from lower energy towards higher energy. Such a group of eigen states, to which a set of vibrational quantum numbers are assigned, was called a feature state.

\section{Hierarchical-Level Structure and Stepwise IVR}

The investigation of the high energy region of acetylene by the DF spectroscopy at low resolution $\left(\sim 10 \mathrm{~cm}^{-1}\right)$ and stimulated emission pumping (SEP) spectroscopy at high resolution $\left(\sim 0.5 \mathrm{~cm}^{-1}\right)$ showed that each feature state contains sub-feature states underneath, and sub-feature states in turn contains a sub-sub-feature states underneath. ${ }^{4}$ This hierarchical level structure occurs because of a stepwise energy flow from an initially populated feature state towards individual eigenstates. That is, the energy flows from an initially localized $\mathrm{CC}$ stretch and trans-bend combination levels into other modes on at least two distinct timescales in the high energy region. Therefore, by assigning the feature states and their underlying finer structure, it becomes possible to specify the energy flow pathway as well as its rate. This explicitly described and characterized energy flow process is the phenomenon of intramolecular vibrational energy redistribution (IVR).

Recently the stepwise IVR pathway was discussed based on detailed examination of the $7000 \mathrm{~cm}^{-1}$ region by SEP spectroscopy. ${ }^{5,6}$ The high-resolution SEP spectrum at lower vibrational energy clearly proved the existence of the DD resonance between the two degenerate bending modes, which plays a crucial role in the earliest time IVR in the highly excited region, and also conclusively identified the importance of additional resonances, i.e. the vibrational $l$-resonance and the 2345 Fermi resonance, which were predicted from IR and Raman spectroscopy ${ }^{15}$ based on the hierarchical structure of the DF spectrum. These resonances promote IVR into the $v_{3}{ }^{\prime \prime}$ mode from the $v_{2}{ }^{\prime \prime}, v_{4}{ }^{\prime \prime}$ and $v_{5}{ }^{\prime \prime}$ modes. Due to this combination of anharmonic resonances which are identified in the high resolution SEP spectrum at low energy, acetylene is destined to be strongly and specifically perturbed at high energy. The essential characteristics of the perturbation at high energy can be predicted by building a Hamiltonian matrix, which incorporates all the anharmonic resonances identified in the lower energy region. 


\section{STATISTICAL ANALYSIS}

There is another type of analysis of the observed complex spectrum, which is known as a statistical analysis, in which statistical properties of the spectrum, i.e. the level spacing and intensity distribution, are treated. Statistical procedures to characterize complex observed spectra were first applied in nuclear physics and is now widely applied to complex spectra of atomic and molecular systems. ${ }^{16}$ Even when a spectrum is formidably complex and no systematic analysis such as that based on assignment quantum numbers is applicable, some characteristic properties of a spectrum such as short- and long-range spectral correlations and intensity distributions can be obtained by a statistical treatment of spectrum.

The complex vibrational level structure of highly excited acetylene was first observed by Abramson et al. ${ }^{7-9}$ in the SEP spectrum. By using the second and third moments of the nearest-neighbor level spacing (NNLS) distribution, a strong correlation among the levels inferred from the incompletely resolved spectrum was identified.

Pique et al. ${ }^{10}$ further investigated vibrationally highly excited acetylene in the energy region around $26500 \mathrm{~cm}^{-1}$ by SEP spectroscopy with $0.3 \mathrm{~cm}^{-1}$ resolution and found a very complex clump structure. When the spectral resolution was improved to $0.05 \mathrm{~cm}^{-1}$, they found an additional level of hierarchical clump structure. In place of assignments of individual peaks in observed clumps, an analysis using a statistical Fourier transform (SFT) ${ }^{11}$ procedure was performed. The SFT analysis showed that there are two time scales ( 3 and $45 \mathrm{ps)}$ ) corresponding to the two stages of the clump structure. By using this statistical procedure, the short range spectral correlation, which can also be extracted from a NNLS distribution, as well as from the spectral intensity distribution, are simultaneously treated and reinterpreted as dynamical behavior. Contrary to the assertions in ref. 10, it has been found that the ability of the SFT to reflect the underlying statistics of the level/intensity distribution is substantially degraded by incomplete resolution. ${ }^{17,18}$ The SFT of the SEP spectrum was further used to propose the existence of a large amplitude vibrational motion, ${ }^{12}$ i.e. a rotational motion of one $\mathrm{H}$ atom around a $\mathrm{CCH}$ core, which is related to the isomerization reaction from acetylene to vinylidene. The isomerization to vinylidene was further inferred from cross-correlation analysis of SEP spectra by Chen et al. ${ }^{13-14}$ This isomerization reaction inferred from the statistical treatments may have a close relationship with the feature state formed by the DD resonance between two degenerate bending modes. According to recent calculations, the lowest member of the $\mathrm{v}_{\text {bend }}=16$ DD resonance polyad has a local mode character with nodal surfaces approximately perpendicular to the acetylene-vinylidene isomerization coordinate (i.e. the "local bender" heads straight towards vinylifene).${ }^{19}$

Though these statistical analyses of SEP spectra of highly excited acetylene strongly stimulated further experimental efforts (mentioned in Sec. II) towards an understanding of the dynamics in highly excited acetylene, it is hard to extract the origin of the correlation or complexity only from the statistical analysis. Only when the statistical information is related to molecular properties such as fundamental vibrational frequencies, can these statistical methods afford meaningful results. 
Recently Coy, Chasman, and Field ${ }^{17}$ applied a new statistical method, an extended auto-correlation (XAC) analysis, to the DF spectrum of acetylene and showed that the anharmonic resonances identified by the generalized conventional spectroscopy in Sec. II can also be extracted but not identified. They showed that contrary to widespread belief, level statistics and the SFT are degraded towards the chaotic limit by poor resolution. They introduced this method as an efficient and secure pattern recognition technique for the automatic identification of repeated, overlapping patterns in congested spectra. The applicability of the XAC method may be demonstrated more clearly, if it successfully derives dynamical information from the unsolved complex spectrum such as the LIF spectrum of $\mathrm{NO}_{2}$.

\section{HIERARCHICAL ANALYSIS}

As described in Sec. II, by comparing low-resolution and high-resolution spectra at higher energy region, the stepwise IVR pathway can be deduced via assignments of the feature states and their underlying substructure. Since the information about the IVR is encoded in the hierarchical structure of the spectrum, dynamical information can be extracted by a convolution analysis of the high resolution spectrum, in which the number of resolved peaks is plotted as a function of the convolution width. When we assign feature states and sub-feature state associated with various levels of hierarchy, we specify the stepwise energy flow pathway. The convolution analysis is, in principle, the same as the tree analysis proposed by Davis, ${ }^{21,22}$ which was recently applied to the LIF spectrum of $\mathrm{NO}_{2}$ by Davis ${ }^{22}$ and by Coy, Chasman, and Field. ${ }^{20}$

Here we apply the convolution analysis to the observed DF spectrum of acetylene in the energy region between 17500 and $19000 \mathrm{~cm}^{-1}$ to demonstrate how this method extracts dynamical information about vibrationally highly excited acetylene. Within that energy range, there are three feature states, $\left(\mathrm{v}_{2}{ }^{\prime \prime}, \mathrm{v}_{4}{ }^{\prime \prime}\right)=(4,16),(5,14),(6,12)$, representing three initially excited FC bright states. The results of the convolution analysis are shown in Figure 1. A naive model based on strong $\Delta_{\mathrm{v}^{\prime}} 4=-\Delta_{\mathrm{v}^{\prime}} 5= \pm 2$ DD resonance and neglect of all other interactions leads one to expect a total of 24 DF peaks in three groups ( 9 for $\mathrm{v}_{\text {bend }}=16,8$ for $\mathrm{v}_{\text {bend }}=14$, and 7 for $\mathrm{v}_{\text {bend }}=12$ ). The plateau found at the convolution width below $\sim 30 \mathrm{~cm}^{-1}$ clearly shows that the feature states caused by the DD resonance represent the first step of the IVR. The small step at a convolution width of $\sim 150 \mathrm{~cm}^{-1}$ simply represents that in this energy range, there are three $\mathrm{FC}$ bright zero-order states and $150 \mathrm{~cm}^{-1}$ is greater than the separation between adjacent sub-features of a single FC bright state. The plateau found above the convolution width of $\sim 250 \mathrm{~cm}^{-1}$ reflects the fact that there is only one broad structure when the convolution width is large enough relative to the energy region sampled in the spectrum.

The above convolution analysis clearly shows that the dynamical information about the first step of IVR, derived from the DF spectrum recorded with a resolution $\sim 10 \mathrm{~cm}^{-1}$, is the energy flow caused by the DD resonance out of the bright zero-order feature states. The convolution analysis also shows that this kind of convolution should be performed while the spectrum is being vibrationally 


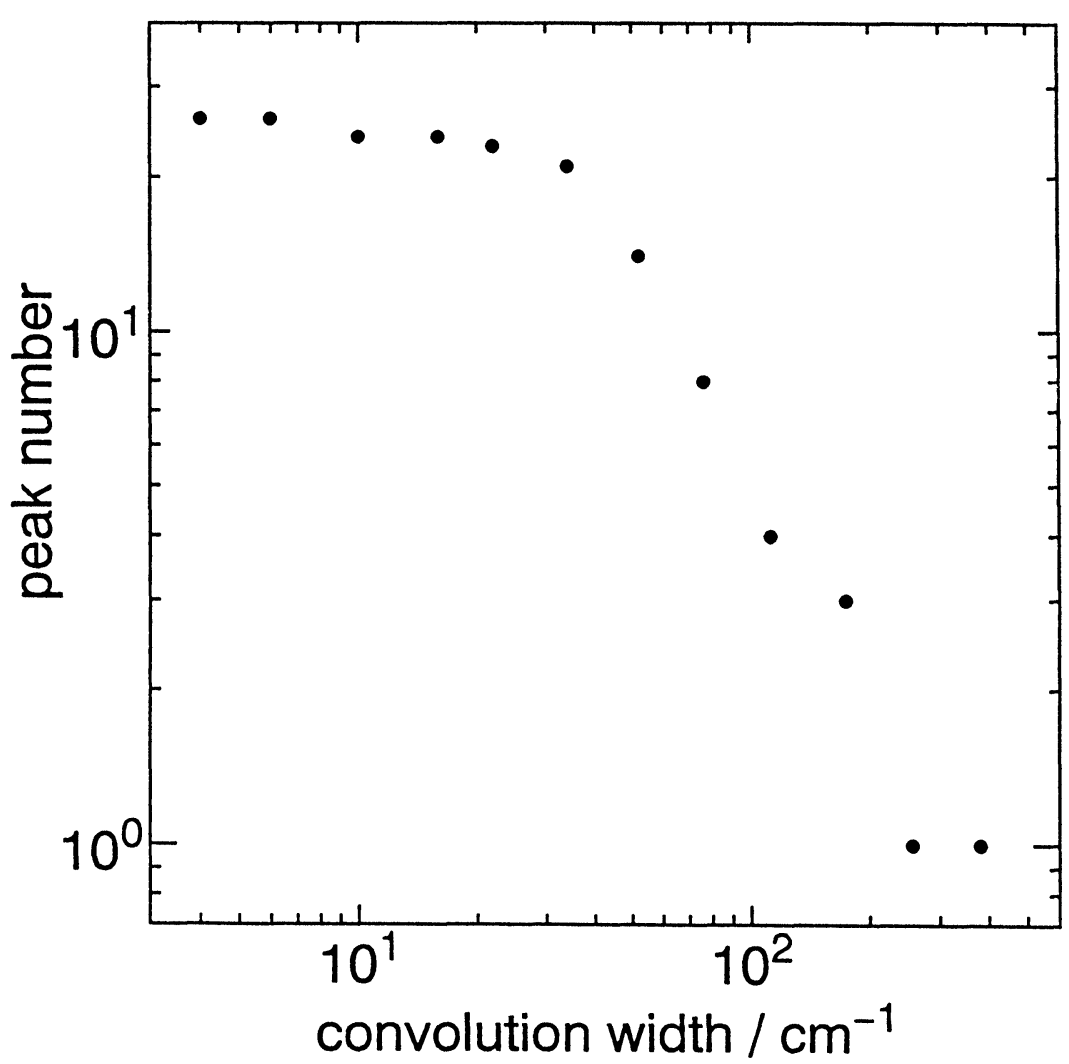

Figure 1 The results of the convolution analysis of the observed DF spectrum ${ }^{4}$ of acetylene recorded by exciting the $V_{0}^{2} K_{0}^{1}$ vibrational band of the $\widetilde{\mathrm{A}}^{1} \mathrm{~A}_{\mathrm{u}}-\widetilde{X}^{1} \Sigma_{\mathrm{g}}^{+}$transition of acetylene in the energy region between 17500 and $19000 \mathrm{~cm}^{-1}$, where $\mathrm{V}$ represents the trans-bending mode $\left(v_{3}^{\prime}\right.$ in the $\tilde{\mathrm{A}}$ state and $v_{4}^{\prime \prime}$ in the $\widetilde{X}$ state). Within this energy range, there are three FC bright feature states, $\left(v_{2}{ }^{\prime \prime}, v_{4}{ }^{\prime \prime}\right)=(4,16)$, $(5,14),(6,12)$. The plateau at the convolution width below $\sim 30 \mathrm{~cm}^{-1}$ clearly shows that the feature states caused by the DD resonance represent the first step of the IVR.

assigned. Otherwise we may misunderstand the number of FC bright feature states, which is not related to the IVR dynamics, as a source of significant information about IVR. The splitting into three FC bright states does contain information about separable normal mode dynamics.

\section{PROCEDURE TO EXTRACT DYNAMICS FROM A COMPLEX SPECTRUM}

From the spectral analyses described above, we can learn the most efficient and systematic procedure to extract the characteristic dynamics of a molecular system encoded in a complex spectrum. As a first step, we should try to find good quantum numbers assignable to feature states at the highest level of the hierarchy. After finding 
some good quantum numbers in the short time dynamics, the resolution of the spectrum should be improved gradually to identify good quantum numbers for the lower level hierarchy, and consequently, to specify the stepwise energy flow pathway from the initially excited FC bright feature states.

In other words, by doing "assigning quantum numbers", which is the essential characteristic of conventional procedure in molecular spectroscopy, to feature states in the spectrum with higher resolution, finer details of the energy flow pathway and rates can be identified and quantified. When we reach a stage in the hierarchy of finer feature states at which definite assignment of individual peaks becomes impossible, a statistical analysis can be performed to determine the extent of mixing of the $n$-th order states, i.e. the extent of IVR.

As mentioned previously, the tree analysis was applied to the complex LIF spectrum of $\mathrm{NO}_{2}$ by Davis ${ }^{22}$ and Coy, Chasman, and Field. ${ }^{20}$ However, the specific energy flow pathway was not derived from the tree analysis of the observed spectrum. Recently, Miyawaki, Yamanouchi and Tsuchiya ${ }^{23}$ recorded the LIF spectrum of $\mathrm{NO}_{2}$ spanning a broad energy range up to the dissociation threshold and assigned feature states of the highest level of the hierarchy based on the convolution analysis. It was shown that IVR proceeds mainly in two steps: i.e. the first step of IVR is an energy flow from the initially prepared feature states with the $v_{2}$ bending mode character in the ${ }^{2} B_{2}$ electronically excited state towards almost all the vibrational feature states within ${ }^{2} \mathrm{~B}_{2}$ manifold and the second step is energy flow from the ${ }^{2} \mathrm{~B}_{2}$ feature states towards vibronic eigenstates via vibronic coupling between the ${ }^{2} \mathrm{~B}_{2}$ state and the vibrationally highly excited region of the ${ }^{2} \mathrm{~A}_{1}$ state. This example also demonstrates that the spectroscopic assignment at the highest hierarchy is critical to specify the energy flow pathway from a complex observed spectrum of a molecular system.

\section{Acknowledgements}

The authors express their thanks to Professor K. Kaya for providing them an opportunity to contribute to this special issue of Laser Chemistry in honor of Professor Mitsuo Ito, Emeritus Professor of Tohoku University, who stimulated their interest in excited state dynamics of polyatomic molecules. K. Y. is grateful to S.L. Coy for providing him with a preprint on the XAC analysis prior to publication.

\section{References}

1. K. Yamanouchi, H. Yamada, and S. Tsuchiya. J. Chem. Phys., 88, 4664 (1988).

2. K. Yamanouchi, S. Takeuchi, and S. Tsuchiya. J. Chem. Phys., 92, 4044 (1990).

3. K. Yamanouchi, in "Molecular Dynamics and Spectroscopy by SEP' edited by H. -L. Dai and R. W. Field. World Scientific (1993).

4. K. Yamanouchi, N. Ikeda, S. Tsuchiya, D. M. Jonas, J. K. Lundberg, G. W. Adamson, and R. W. Field. J. Chem. Phys., 95, 6330 (1991).

5. D. M. Jonas, S. A. B. Solina, B. Rajaram, R. J. Silbey, R. W. Field, K. Yamanouchi, and S. Tsuchiya. J. Chem. Phys., 97, 2813 (1992).

6. D. M. Jonas, S. A. B. Solina, B. Rajaram, R. J. Silbey, R. W. Field, K. Yamanouchi, and S. Tsuchiya. J. Chem. Phys., in press. 
7. E. Abramson, R. W. Field, D. Imre, K. K. Innes, J. L. Kinsey. J. Chem. Phys., 80, 2298 (1984).

8. E. Abramson, R. W. Field, D. Imre, K. K. Innes, J. L. Kinsey. J. Chem. Phys., 83, 453 (1985).

9. R. L. Sundberg, E. Abramson, J. L. Kinsey and R. W. Field. J. Chem. Phys., 83, 466 (1985).

10. J. P Pique, Y. Chen, R. W. Field, and J. L. Kinsey. Phys. Rev. Lett., 58, 475 (1987).

11. L. Leviandier, M. Lombardi, R. Jost, and J. P. Pique. Phys. Rev. Lett., 56, 2449 (1986).

12. J. P. Pique, M. Lombardi, Y. Chen, R. W. Field, and J. L. Kinsey Ber. Bunsenges. Phys. Chem., 92, 422 (1988).

13. Y. Chen, D. M. Jonas, C. E. Hamilton, P. G. Green, J. L. Kinsey, and R. W. Field. Ber. Bunsenges. Phys. Chem., 92, 329 (1988).

14. Y. Chen, D. M. Jonas, J. L. Kinsey, and R. W. Field. J. Chem. Phys., 91, 3976 (1989).

15. (a) J. Pliva. J. Mol. Spectrosc. 44, 145 (1972), (b) 44, 165 (1972).

16. F. Haake, "Quantum Signatures of Chaos", Springer-Verlag (1991).

17. J. Wllkie and P. Brumer. Phys. Rev. Lett., 69, 2018 (1992).

18. J. P. Pique, Y. Chen, R. W. Field, and J. L. Kinsey. Phys. Rev. Lett., 69, 2019 (1992).

19. E. L. Sibert and R. C. Mayrhofer. J. Chem. Phys., 99, 937 (1993).

20. S. L. Coy, D. Chasman, and R. W. Field, in "Molecular Dynamics and Spectroscopy by SEP" edited by H. -L. Dai and R. W. Field, World Scientific (1993).

21. M. J. Davis. J. Chem. Phys. Lett., 192, 479 (1992).

22. M. J. Davis. J. Chem. Phys., 98, 2614 (1993).

23. J. Miyawaki, K. Yamanouchi, and S. Tsuchiya, to be submitted. 